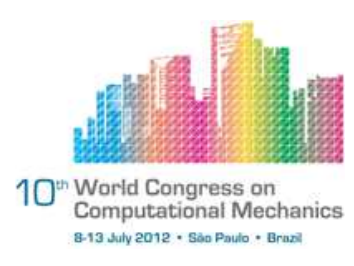

\title{
ORIENTATION OPTIMIZATION FOR THE FLUTTER ONSET IN FIBER REINFORCED LAMINATED PLATES USING MAC
}

\author{
G. Varella ${ }^{1}$, D. M. De Leon ${ }^{1}$, J. S. O. Fonseca ${ }^{1}$, C. E de Souza ${ }^{2}$, R. G. A. da Silva ${ }^{2}$ \\ ${ }^{1}$ Applied Mechanics Group, Engineering School at the Federal University of Rio Grande do \\ Sul - Porto Alegre - RS - Brazil (jun@ufrgs.br) \\ ${ }^{2}$ Aerodynamics Department, Technological Institute of Aeronautics - São José dos Campos - \\ SP - Brazil
}

\begin{abstract}
This work presents an optimization formulation to increase the flutter onset velocity in a composite plate made of glass-epoxy subject to an airflow parallel to its surface. The problem is stated as the maximization of the eigenvalue related to the flutter effects, aiming the improvement in the flutter onset speed. The design variable is the fiber orientation of each ply of the composite plate. The sensitivities are calculated analytically and sequential linear programming is applied. The flutter mode and onset velocity are calculated by the ZAERO software. Mode tracking switching scheme based on the modal assurance criterion (MAC) is used to improve the optimization formulation when a repeated eigenvalue problem has to be handled by the algorithm. Results are presented for many test cases, showing a marked improvement of the simulated flutter behavior.
\end{abstract}

Keywords: Structural Optimization, Aeroelasticity, Composite Flat Plates, Modal Assurance Criterion.

\section{INTRODUCTION}

The development of fibrous composite materials are tightly related to its use in aeronautic industry. Characteristics such as lightness and high strength were rapidly employed in the construction of airplanes, sailplanes, micro-air vehicles and so on. Once the first sailplane using fiberglass was presented in West Germany [14] the study of composite materials in aeronautic construction became almost mandatory, nowadays the use of composite materials in aeronautic design is responsible for $15 \%$ of structural weight of civil aircrafts and more than $50 \%$ of structural weight in helicopters and military aircrafts [5], in space applications these numbers are still bigger.

A special chapter of composite materials application in aircrafts design is its use in unmanned aerial vehicle (UAV). Although its first use was in military applications, these kind of vehicles have been widely used in civilian applications in the last decades; mainly in electric lines inspection, aerial images to farmers, car traffic observation, environmental monitoring 
and so on. Beside the safety and easiness of controlling these devices, its high relation thrustpayload gives to these small aircrafts the capability of flying higher, faster and for longer time with less fuel, turning they environmentally friendly options. An important characteristic of UAS wings is the high displacement they are subject, much more than the bigger aircrafts with rigid wings. These wings can show LCO during normal operation parameters. LCO stands for Limit Cycle Oscillation and it can be described as a high amplitude oscillation without frequency and amplitude changes in the absence of external perturbations. In aircrafts design this kind of aeroelastic behavior must be avoided, it can lead to effects like structural fatigue, coupling of control system frequencies, etc. Excepting the nonlinear nature of the coupling of structural response with non-stationary aerodynamic forces, the LCO frequency and onset speed can be related to the aeroelastic stability analysis in the calculation of flutter speed [3]. Therefore, the linear analysis used for flutter characteristics study can also predict the onset of the LCO phenomenon.

The linear theory indicates a limit dynamic load which the plate or shell movements become unstable and grow in time. Such effects are known in general as flutter and depend on coupling two or more modes whose oscillations create aerodynamic forces that allow energy to be transferred from the air-stream to the structure [15].

At the same time that the application of low aspect ratio composite wings in aircraft designs increased the likelihood of aeroelastic undesirable effects, its different properties in different directions gave the designer an important tool to deal with these phenomena. This problem emphasizes the importance of structural optimization role in the laminated composite wings design. Nowadays, the development of this area increased in such manner that it became an important branch of aeroelastic design. This area is known as aeroelastic tailoring and can be defined as the design process that makes use of the directional properties of fibrous composite materials in wing skins and orients these materials in optimum directions [8]. Then aeroelastic tailoring must be done with special attention to optimization methods, not only to aeroelastic analysis methods.

This work presents an aeroelastic tailoring of a glass-epoxy flat plate wing immersed in a subsonic flow, aiming an increase in the flutter speed onset. The design variable of the optimization problem is the flat plate fibrous direction of each ply, the objective function is the maximization of the eigenfrequency related to the flutter effects onset. The analysis is split in aeroelastic part and structural part, and each one is solved by different means and coupled by the use of splines. The aeroelastic part is accomplished by using the ZAERO software that solves the generalized equation of motion for the wing immersed in a subsonic flow parallel to its surface. The aeroelastic stability analysis results in the flutter speed onset and the associated wing structural mode that causes the flutter effects. Having the structural mode and its eigenfrequency the structural part is developed using Matlab software, the finite element method (FEM) with a serendipity shell element is applied. The algorithm is written as the maximization of the flat plate wing eigenfrequency sought in aerodynamic analysis, it uses sequential linear programming (SLP) as the search method and the sensitivities of the objective function related to the design variable is calculated analytically. In order to improve the formulation, a mode tracking switching scheme based on the modal assurance criterion (MAC) [2] and [11] is applied to deal with the repeated eigenvalue problem when 
it is necessary. The flat plate wing structural analysis uses the first-order shear deformation theory (FSDT), although FSDT is derived for general composites, in this work only directional fabric is used, in order to emphasize the fibrous orientation importance in the design.

\section{STRUCTURAL ANALYSIS}

The structural analysis is performed by the finite element method (FEM). The Ahmad degenerated shell element is applied, this element is a well-known finite element for the treatment of shell and plates structures. A complete review of this element can be found in Zienkiewicz [17].

In this work the First-Order Shear Deformation Theory (FSDT) is applied, comparing with the Classical Lamination Theory the FSDT provides better relation between computational efficiency and accuracy of the global structural behavior [16]. In the FSDT the constitutive equation of laminate plate is described as:

$$
\left\{\begin{array}{l}
\mathbf{N} \\
\mathbf{M}
\end{array}\right\}=\left[\begin{array}{ll}
\mathbf{A} & \mathbf{B} \\
\mathbf{B} & \mathbf{D}
\end{array}\right]\left\{\begin{array}{c}
\varepsilon \\
\kappa
\end{array}\right\},
$$

where $\mathbf{N}$ and $\mathbf{M}$ are the distributed tractions and moments, respectively, applied to the plate, the terms in $\varepsilon$ are midplane (membrane) strains, and in $\kappa$ are curvatures, second derivatives of the transverse displacements.

The sub-matrices A, B and D are the extensional stiffness matrix, the coupling stiffness matrix and the bending stiffness matrix, respectively. The matrix $\mathbf{B}$ has the feature of differing from zero when the plate is not symmetric. Each matrix can be described as follow:

$$
\begin{gathered}
\mathbf{A}=\sum_{k=1}^{n p} \overline{\mathbf{Q}}\left(z_{k+1}-z_{k}\right), \\
\mathbf{B}=\frac{1}{2} \sum_{k=1}^{n p} \overline{\mathbf{Q}}\left(z_{k+1}^{2}-z_{k}^{2}\right), \\
\mathbf{D}=\frac{1}{3} \sum_{k=1}^{n p} \overline{\mathbf{Q}}\left(z_{k+1}^{3}-z_{k}^{3}\right),
\end{gathered}
$$

where $z_{k}$ is the distance from laminate midplane to the bottom of the $k$-th ply, $\overline{\mathbf{Q}}$ is the transformed stiffness matrix, and $n p$ the number of plies.

A complete discussion about composite materials and the theory presented above can be found in the textbooks of Jones [10] and Reddy [13].

Once the constitutive relations for composite laminated materials are determined, the mass and stiffness matrices can be assembled. Kumar and Palaninathan presented a formulation for the stiffness matrix assembly in which the inverse isoparametric mapping Jacobian matrix is considered constant, the computations are carried only on the reference surface [12].

Considering the stiffness matrix can be described as

$$
\mathbf{K}_{e}=\int_{\Omega_{e}} \mathbf{F}^{T} \mathbf{C F} \Omega_{e}
$$


where $e$ is related to the finite element, the matrix $\mathbf{F}$ is rewritten as

$$
\mathbf{F}=\mathbf{F}_{1}+z \mathbf{F}_{2}
$$

the stiffness matrix can be now expressed as

$$
\mathbf{K}_{e}=\int_{-1}^{+1} \int_{-1}^{+1}\left[\mathbf{F}_{1}^{T} \mathbf{A} \mathbf{F}_{1}+\mathbf{F}_{1}^{T} \mathbf{B} \mathbf{F}_{2}+\mathbf{F}_{2}^{T} \mathbf{B} \mathbf{F}_{1}+\mathbf{F}_{2}^{T} \mathbf{D} \mathbf{F}_{2}\right]|J| \eta \xi
$$

where the matrices $\mathbf{A}, \mathbf{B}$ and $\mathbf{D}$ have already been defined.

The lumped mass matrix assembled in this work can be described as

$$
\mathbf{M}_{e}=\int_{\Omega_{e}} \mathbf{N}^{T} \gamma \mathbf{N} \Omega_{e}
$$

where $\mathbf{N}$ are the interpolation functions for Ahmad element, and

$$
\left\{\begin{array}{ccc}
N_{i} \gamma N_{j} & \text { if } & i=j \\
0 & \text { if } & i \neq j
\end{array},\right.
$$

where the terms related to the slopes are assumed null.

The global mass and stiffness matrices are assembled from the corresponding elemental matrices. For free undamped vibrations the equation of motion is:

$$
\mathbf{M} \ddot{\mathbf{q}}+\mathbf{K q}=0 .
$$

After applying some standard procedures, the equation 10 can be written as the wellknown generalized eigenvalue problem,

$$
\left(\mathbf{K}-\lambda_{i} \mathbf{M}\right) \Phi_{i}=0 .
$$

Applying the the boundary conditions, equation 11 can be solved numerically for a chosen number of eigenvalues and their associated eigenvectors.

In the algorithm applied in the work, the number of modes involved in the modal analysis is chosen by a effective modal mass calculation [9]. It is done in order to determining which structural modes are effectively being important in vibration analysis.

\section{AEROELASTIC ANALYSIS}

Aeroelasticity is the design activity that study the behavior of structures subject to aerodynamic loads and structural deformation acting together. The changes in the surface shape are due to the pressure field variations caused by interaction fluid/structure. The term aeroelasticity was introduced by Cox and Pugsley in the early 30's to call attention to this kind of problem [15]. Then aeroelasticity comprises a serie of disciplines working together as aerodynamics, structures and inertia.

In this work the analysis of interest is the structure stability of a composite flat plate wing subject to aeroelasticity efforts, and supposing a linear system the flutter analysis is 
applied. The linear theory indicates a limit dynamic pressure which the shell or plate displacement becomes non-stable and grows in time. Such effects are known as flutter. Flutter is a self-excited dynamic instability. It depends on the coupling of two or more vibrations modes in which its oscillations allow energy to be transferred from the airstream to the structure and the modes oscillations grow in time. Nevertheless, non-linear effects due to the large oscillatory displacements raise stresses in the plane then effects that stabilizes the structure show up and it is possible to work with speeds higher than the ones predicted in the linear theory. These effects are known as Limit Cycle Oscillation (LCO) and, besides treatable, such oscillatories effects can cause a set of problems like structural fatigue and couple with others modes in the vehicle. Therefore in most cases the prevention of flutter onset is the first project criteria. That is the reason why flutter analysis is accomplished in this work. A complete description about aeroelastic design can be found for instance in the reference book of Weisshaar [15].

\subsection{Foundations of Aeroelasticity}

The aeroelastic response of a vehicle in flight is a result of the interactions among the structural and inertial forces, aerodynamic forces induced by static or dynamic strain of the structure and external loads [18]. The equation of motion can be written as:

$$
\mathbf{M x}+\mathbf{K x}=\mathbf{f},
$$

where $\mathbf{M} \ddot{\mathbf{x}}$ and $\mathbf{K x}$ are the inertial and structural forces respectively. The vector $\mathbf{f}$ means the aerodynamic forces applied to the structure and can be divided into two parts, a part of aeodynamic load induced by structural strains and other due to external loads such as wind gusts and control:

$$
\mathbf{f}=\mathbf{f}_{a}(\mathbf{x})+\mathbf{f}_{e}(t),
$$

where $\mathbf{f}_{a}(\mathbf{x})$ represents the aerodynamic forces induced by structural strains and $\mathbf{f}_{e}(t)$ the external forces. As the first part depends on the structural strains $\mathbf{x}(t)$ this relation can be interpreted as an aerodynamic feedback. Using this concept, the equation 13 can be rewritten as

$$
\mathbf{M} \ddot{\mathbf{x}}+\mathbf{K x}-\mathbf{f}_{a}(\mathbf{x})=\mathbf{f}_{e}(t),
$$

where the left side of the equation 14 is a closed dynamic system self-excited by the nonstationary aerodynamic loading [18]. Generally, the flutter analysis leads to the stability search of a structure subject to aeroelastic efforts in terms of flight speed and dynamic pressure. Being $\mathbf{f}_{a}(\mathbf{x})$ a non-linear function with respect to $\mathbf{x}(t)$, the analysis is accomplished by an iterative procedure solving the following equation

$$
\mathbf{M} \ddot{\mathbf{x}}+\mathbf{K x}-\mathbf{f}_{a}(\mathbf{x})=0,
$$

with $\mathbf{x}(0)$ and $\dot{\mathbf{x}}(0)$ as boundary conditions, set in $t=0$. However, this problem resolution requires a tremendous computational effort, once it is just possible by computational dynamics

methods (CFD, Computational Fluid Dynamics). Instead of this, the usual practice in flutter 
analysis is transform the equation 15 into the frequency domain in a way that a non-stationary aerodynamic can be applied, defined by simple harmonic motions in the same domain.

Before the next step, it is important to notice the problem shown in equation [15] is divided in two parts, structural and aerodynamic. The inertial and structural forces in the equation of motion are represented by matrices and vectors identified by the finite element mesh created to the structural model. However the vector and points represented by $\mathbf{f}_{a}(\mathbf{x})$ are defined to set another physical model, in other words, it is likely the aeroelastic and structural model control points do not match.

The issue aforementioned introduces another necessary transformation to make the two models match. The need for representing some physical coordinates into other points is overcome by applying the follow transformation:

$$
\mathbf{x}_{a}=\mathbf{G}_{s} \mathbf{x}_{e}
$$

where $\mathbf{G}_{s}$ is a matrix that interpolates the displacement shape from the structural model to the aerodynamic model, given by splines transformation presented by Harder and Desmarais [7].

Following the analysis, by using a linear modeling the assumption of small displacements is allowed and the superposition principle can be applied. The generalized matrices formulation is then used. For further explanation about the development of structural dynamic model in the modal basis the authors refer the Zaero manual [18].

The structural displacement can be approximated by the relation:

$$
\mathbf{x}=\Phi \overline{\mathbf{x}}
$$

where $\overline{\mathrm{x}}$ is the vector of generalized displacements and $\Phi$ is a matrix containing the eigenvectors on each column from the modal analysis of the structural model.

One can write the undamped equation of motion based on the previous explanation like this:

$$
\boldsymbol{\Phi}^{T} \mathbf{M} \Phi \overline{\ddot{x}}+\boldsymbol{\Phi}^{T} \mathbf{K} \Phi \bar{x}=\boldsymbol{\Phi}^{T} \mathbf{f}
$$

that can be rewritten as:

$$
\tilde{\mathbf{M}} \overline{\ddot{x}}+\tilde{\mathbf{K}} \overline{\mathbf{x}}=\Phi^{T} \mathbf{f}
$$

where $\tilde{\mathbf{M}}$ and $\tilde{\mathbf{K}}$ are the mass and stiffness generalized matrices respectively.

\subsection{Aerodynamic Model}

At the end of the 1960's, Albano e Rodden [1] presented a description of the Doublet Lattice Method (DLM) for analysing lifting surface loading in unsteady flow. Later, Giesing et al. [6] show an expansion to non-planar surfaces, along with a detailed method description. For the present work the aeroelastic analysis is done by means of Zaero software, that uses the Zona6 Method, a DLM variation.

In the DLM, the lifting surface is discretized in small aerodynamic panels. Each panel has a control point where the boundary conditions are applied. The panels are treated equally, 
regardless their wing bound distance. The dipole line is placed at $\frac{1}{4}$ of the chord length of each panel and the displacement is calculated at $\frac{3}{4}$ of the chord length. The figure 1 shows a wing discretized into doublet lattice panels, and in detail a single panel with doublet line and a collocation point.

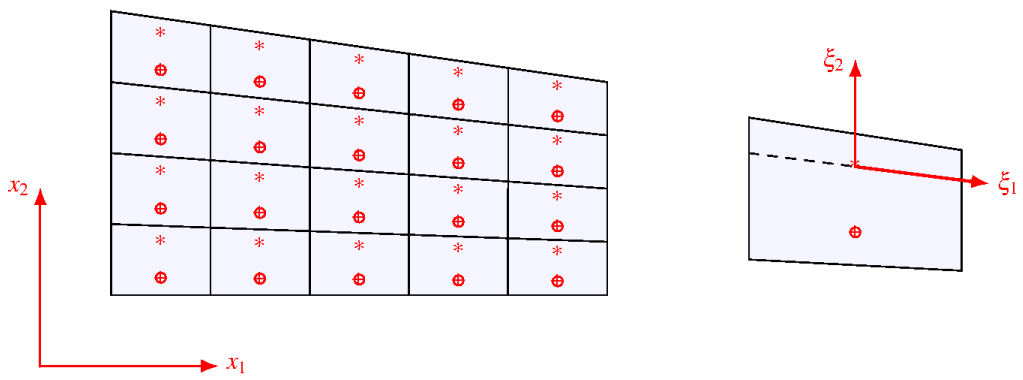

Figure 1. Panel method with control points.

Considering the aerodynamic control points displacements as $\mathbf{q}_{a}$, a vector containing the points, the aerodynamic forces that act in these aerodynamic panels as a function of these displacements can be defined as:

$$
\mathbf{f}_{a}=q_{\infty} \mathbf{A}(i k) \mathbf{q}_{a}
$$

where $q_{\infty}$, is the dynamic pressure, $k=\omega b / U$, is the reduced frequency, $\omega$, is the harmonic frequency, $U$, is the free stream velocity, and $b$ is the reference semi-chord. The aerodynamic influence coefficient matrix $\mathbf{A}(i k)$, is a function of the reduced frequency.

Therefore, the generalized aerodynamic forces vector is given by:

$$
\tilde{\mathbf{f}}=\mathbf{\Phi} \mathbf{G}_{s} \mathbf{f}_{a}=q_{\infty} \mathbf{\Phi} \mathbf{G}_{s}^{T} \mathbf{A}(i k) \mathbf{q}_{a}
$$

The equation 21 is not ready to be placed in equation 19 yet, the terms are still written in the aerodynamic control points. Writing the equation 21 in terms of MEF points it is necessary switch the vector containing aerodynamic displacements as

$$
\mathbf{q}_{a}=\Phi_{a} \Upsilon=\mathbf{G}_{s} \Phi \Upsilon,
$$

making this transformation, the vector can be written in the points described by MEF as

$$
\tilde{\mathbf{f}}=q_{\infty} \boldsymbol{\Phi} \mathbf{G}_{s}^{T} \mathbf{A}(i k) \mathbf{G}_{s} \boldsymbol{\Phi} \Upsilon
$$

This way allows to represent a generalized matrix containing the aerodynamic coefficients of influency $\mathbf{Y}(\mathrm{ik})$ that would be the matrix $\mathbf{A}(\mathrm{ik})$ described in MEF points:

$$
\mathbf{Y}(i k)=\boldsymbol{\Phi}^{T} \mathbf{G}_{s}^{T} \mathbf{A}(i k) \mathbf{G}_{s} \boldsymbol{\Phi} .
$$

Now all the terms can be described in the generalized form, once the both sides of equation 19 have the size of the desired number of modes in the analysis. The equation can be rewritten as:

$$
-w^{2} \tilde{\mathbf{M}} \Upsilon+\tilde{\mathbf{K}} \Upsilon=q_{\infty} \mathbf{Y}(i k) \Upsilon
$$


or separating the variables:

$$
\left[-w^{2} \tilde{\mathbf{M}}+\tilde{\mathbf{K}}-q_{\infty} \mathbf{Y}(i k)\right] \mathbf{\Upsilon}=0 .
$$

The equation 26 is a stability problem with solutions different than the trivial being sought. There are a sort of methods available to solve the problem stated in 26. In this work the g-method presented by Chen [4] is applied by the software Zaero. For further explanation about g-method the authors refer the work of Chen and the Zaero manual [18].

Basically, the method searches for not only the point where the aeroelastic instability occurs, but also the vibration modes and parameters associated with it, such as frequency and damping values. In this work we are interested in the study of flutter onset and the mode associated, it is not the scope of $t$ his work analyse the structural behavior under sub-critical conditions (before flutter onset). The aeroelastic analysis results are presented in the form of VGF curves (velocity-damping-frequency). These graphics show the evolution of the flatplate wing performance in a given airstream range. We are focused in the point where the damping evolution line crosses the zero value, this point indicates the structure is undergoing from a stable condition (negative damping values) to an unstable condition (positive values for damping).

\section{FIBER ORIENTATION OPTIMIZATION}

In this work, we obtain the laminated ply configuration to maximize the eigenvalue related to flutter effects of a cantilevered laminated plate. The chosen eigenfrequency is maximized in an unconstrained formulation, where the fiber orientation of each ply are the design variables, i.e., the number of design variables is the number of plies in the laminated plate.

The optimization problem can be stated as follows:

$$
\text { maximize }\left(\frac{\lambda}{\lambda_{0}}\right)
$$

where $\lambda_{0}$ is the eigenvalue of the initial design, and $\lambda$ is the eigenvalue updated during the optimization process. This formulation allows the algorithm to find the laminate configuration that increases the gap between the eigenvalues whose interaction is causing flutter effects. The goal in analysis is to set the lamination parameters in a way that the interaction that causes flutter effects shows up in higher speeds.

It is important to remark that the optimization formulation takes into account all eigenvalues within a given range. When a root multiplicity is detected, the algorithm uses a mode tracking switching scheme based on the Modal Assurance Criterion (MAC) [11]. This scheme allows the algorithm to run faster and more accurate than the simple repeated eigenvalue problem, to overcome the root multiplicity problem.

In the first step, the discrete eigenvalue problem is solved, and an input file containing eigenvalues and eigenmodes is created. In the second step, the optimization process starts and the objective function is computed. The flowchart is shown in figure 2 .

If the objective function converges, the optimization algorithm stops, otherwise the next step is the computation of eigenvalues sensitivities with respect to design variables. As 


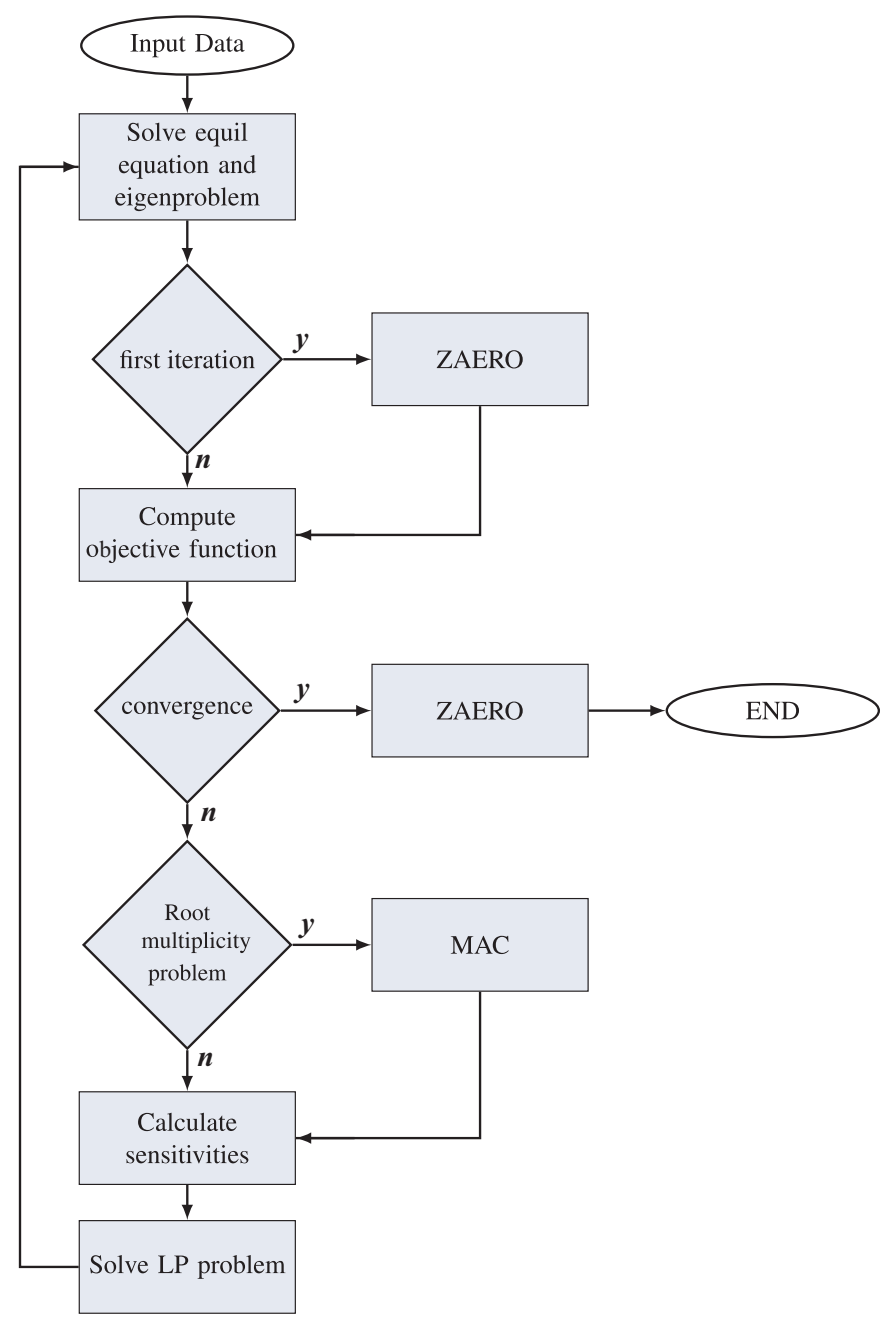

Figure 2. Flowchart of the optimization procedure

final step of the loop, the design variables are updated by the sequential linear programming (SLP). When convergence is reached, a new set of eigenfrequencies and its eigenvectors are obtained for the final aeroelastic analysis of the optimized structure.

The sensitivity of the $j$-th eigenvalue $\lambda_{j}=\omega_{j}^{2}$ with respect to the $i$-th design variable $\theta_{i}$ is obtained by

$$
\frac{\partial \lambda_{j}}{\partial \theta_{i}}=\frac{\boldsymbol{\Phi}_{j}^{T}\left(\frac{\partial \mathbf{K}}{\partial \theta_{i}}-\lambda_{j} \frac{\partial \mathbf{M}}{\partial \theta_{i}}\right) \boldsymbol{\Phi}_{j}}{\boldsymbol{\Phi}_{j}^{T} \mathbf{M} \boldsymbol{\Phi}_{j}}
$$

where $\mathbf{K}$ and $\mathbf{M}$ are the stiffness and mass matrices, respectively. $\boldsymbol{\Phi}_{j}$ is the discretized $j$ eigenmode, and $\theta$ is the fiber angle of composite ply. In this case, is useful to notice that, as the mass of the elements do not vary with the angle of the fibers, so the derivative of $\mathbf{M}$ with respect to $\theta$ is zero. And since the eigenvectors are normalized by mass, the equation 28 becomes

$$
\frac{\partial \lambda_{j}}{\partial \theta_{i}}=\boldsymbol{\Phi}_{j}^{T}\left(\frac{\partial \mathbf{K}}{\partial \theta_{i}}\right) \boldsymbol{\Phi}_{j}
$$




\subsection{Modal Assurance Criterion}

The goal of the work is maximize an eigenvalue related to specific mode, therefore a tracking scheme to monitoring the target mode is important. This can be done by means of a mode tracking scheme like the modal assurance criterion. The MAC goal is to promote a consistent measure (linearity degree) between eigenvectors [2].

The MAC definition is

$$
\operatorname{MAC}\left(\boldsymbol{\Phi}_{a}, \boldsymbol{\Phi}_{b}\right)=\frac{\left|\boldsymbol{\Phi}_{a}^{T} \boldsymbol{\Phi}_{b}\right|^{2}}{\left(\boldsymbol{\Phi}_{a}^{T} \boldsymbol{\Phi}_{a}\right)\left(\boldsymbol{\Phi}_{b}^{T} \boldsymbol{\Phi}_{b}\right)},
$$

where $\Phi_{a}$ and $\Phi_{b}$ are the target modes that must be compared. The MAC values are between 0 and 1 , where 0 indicates inconsistency and values around 1 indicate a good linear relation.

Kim and Kim [11] observed that the reference mode can be updated during the optimization process, if the switch during the iteration is large. Therefore, after the definition of the reference eigenvector, this one is updated as the eigenvector with the MAC nearer 1 on each iteration.

\section{RESULTS}

The model used in this work is shown in figure 3, a graphite-epoxy flat-plate wing with an airstream parallel to its surface. The plate is $0.45 \mathrm{~m}$ length, $0.08 \mathrm{~m}$ width and 0.005 $\mathrm{m}$ of thickness on each ply. Two different models are tested in this work, a non-symmetric plate with two plies and a symmetric plate with four plies. The material density is $\rho=$ $1793\left[\mathrm{Kg} / \mathrm{m}^{3}\right]$. The material properties are shown in table 1 .

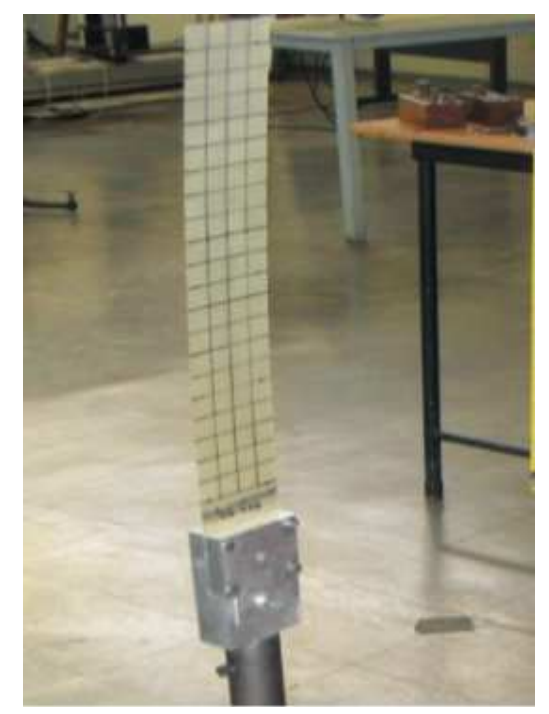

Figure 3. Physical model.

The physical model shown in figure 3 is a non-symmetrical flat-plate and its laminated configuration is $[0,90]$. The data in table 1 and validation for finite element mesh was obtained by means of experimental tests on the physical model. The effective modal mass calculation determines that the first five eigenvectors must be took into account. 
Table 1. Ply properties.

\begin{tabular}{ccc}
\hline$E_{1}=29.090 \mathrm{GPa}$ & $G_{12}=2.726 G P a$ & $\nu_{12}=0.1434$ \\
\hline$E_{2}=8.841 G P a$ & $G_{13}=2.726 G P a$ & $\nu_{13}=0.1434$ \\
\hline$E_{3}=8.841 G P a$ & $G_{23}=1.205 G P a$ & $\nu_{23}=0.2933$ \\
\hline
\end{tabular}

\subsection{Non-symmetric wing}

The first test case is the two plies composite laminated plate, the laminate configuration is $[0,90]$. The modal analysis of the plate is shown in the figure 4.

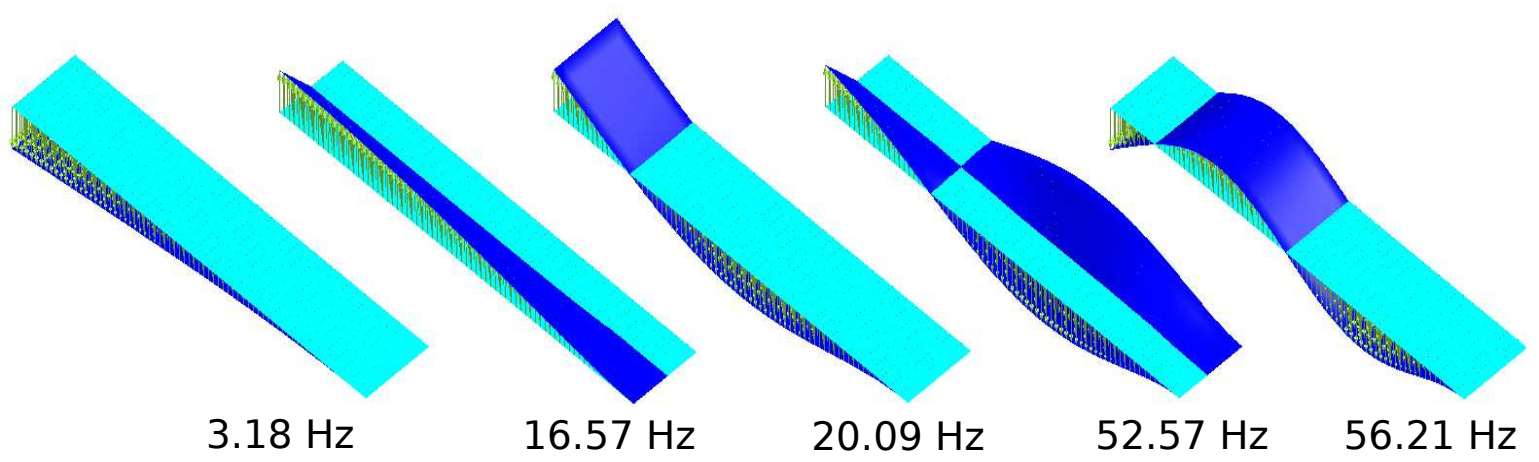

Figure 4. Modal analysis of the $[0,90]$ plate.

The next step in analysis is the wing aeroelastic behavior by means of Zaero software. Using the results in structural modal analysis, the aeroelastic analysis shows the mode responsible for flutter effects and the flutter mechanism as well. The figure 5 shows the flutter mode and figure 6 is the VGF plot of the non-optimized structure. The results show a frequency coalescence between the first and second modes, the second mode (first torsional mode) is the mode that is causing flutter effects, according to the VGF plots.

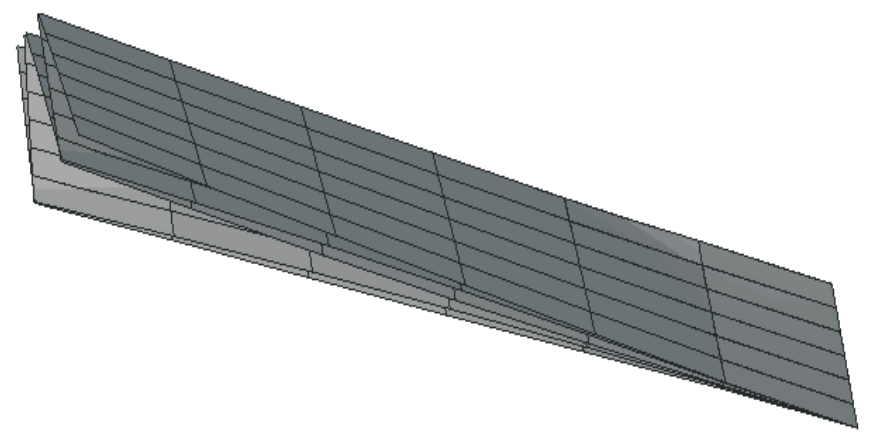

Figure 5. Flutter mode.

The optimization problem is written as the maximization of the second eigenfrequency of the flat-plate wing. The figure7 7 shows the development of the objective function and values for MAC during the optimization. As shown in the figure, during the process the eigenvalue reaches the next one, and a root multiplicity problem has to be solved. According to the MAC evolution, the switching mode problem is solved successfully. 


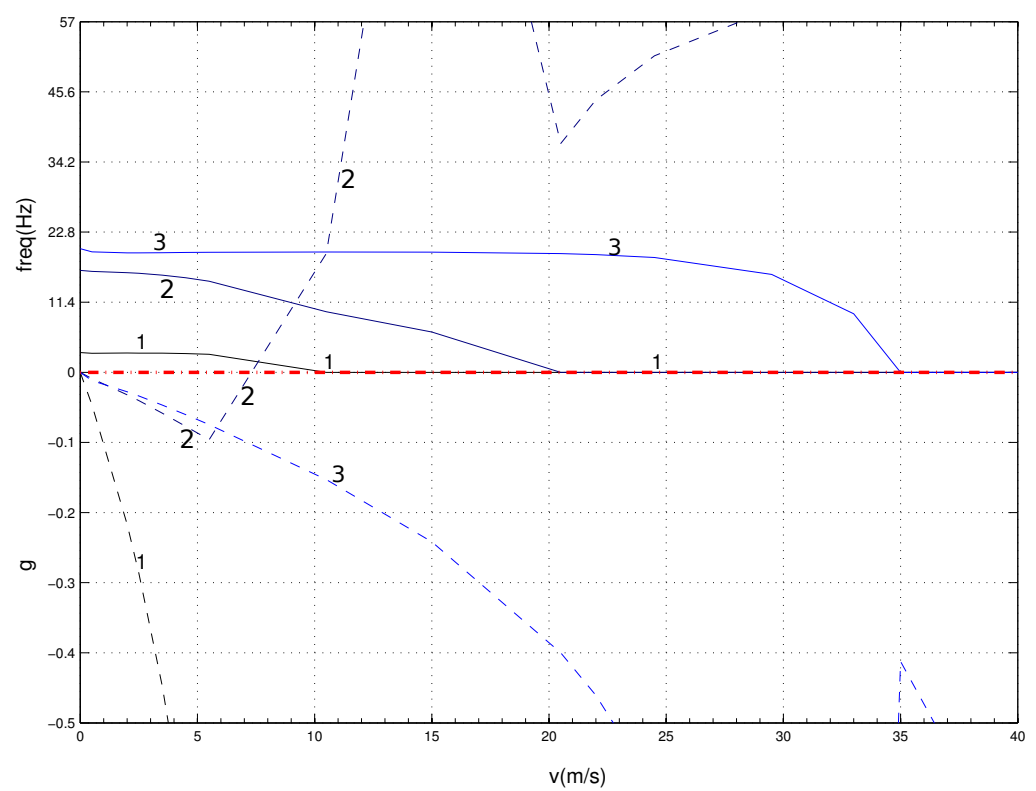

Figure 6. Aeroelastic analysis of the $[0,90]$ plate.

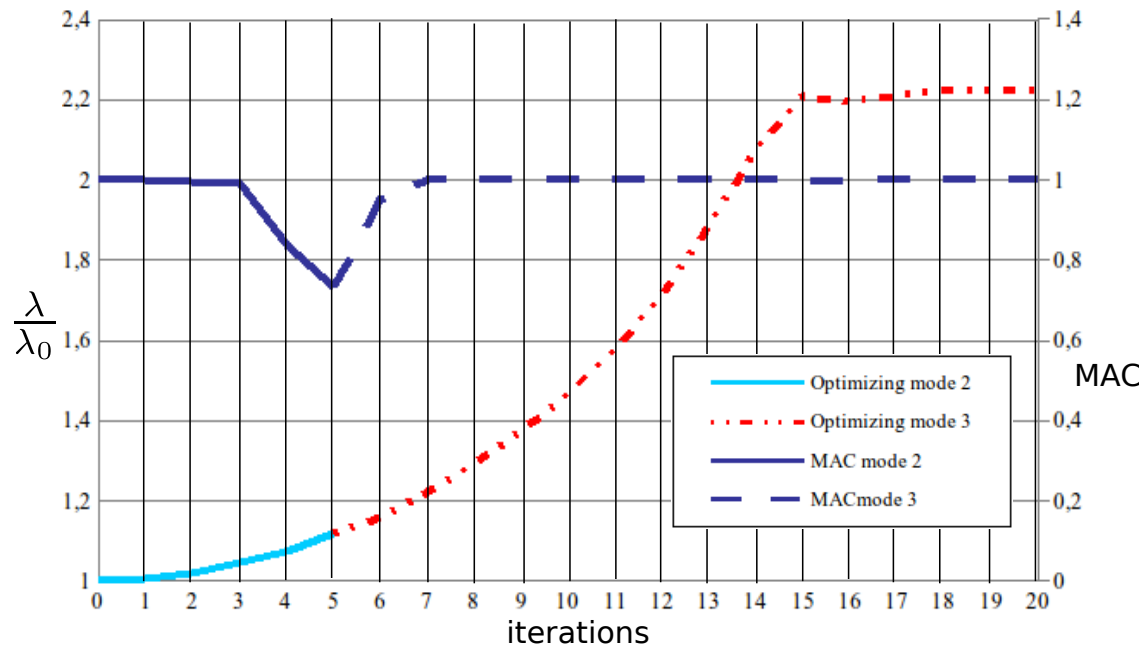

Figure 7. Objective function and MAC evolution.

As aforementioned, the target mode is the first torsional mode, the initial frequency value is $16.57[\mathrm{~Hz}]$ and after the optimization the value of the first torsional mode is 36.87 $[\mathrm{Hz}]$, an increase of $123 \%$. The aeroelastic analysis after the optimization (figure 8) shows an increase of $117 \%$ in flutter speed onset. The table 2 summarizes the results before and after the optimization procedure.

In all VGF graphics only the modes involved in flutter analysis are plotted, although all 5 eigenvectors are included in analysis.

Regarding the model simplicity ( 2 plies), it is possible to plot the third frequency field versus the range of the fiber angles of each ply. The plot results (figure 9) shows that the maximum frequency value is around $35 \mathrm{~Hz}$, when both ply orientations are around 53 degrees. The results agree with those found in the optimization procedure. 


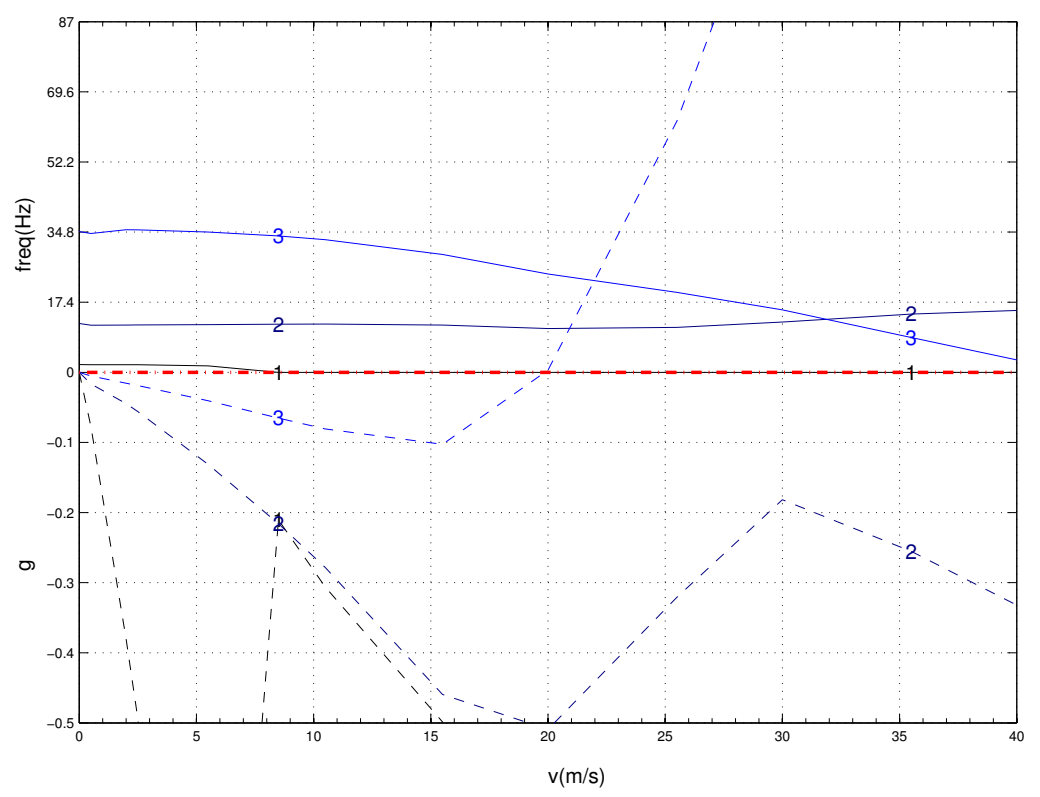

Figure 8. VGF plot after optimization.

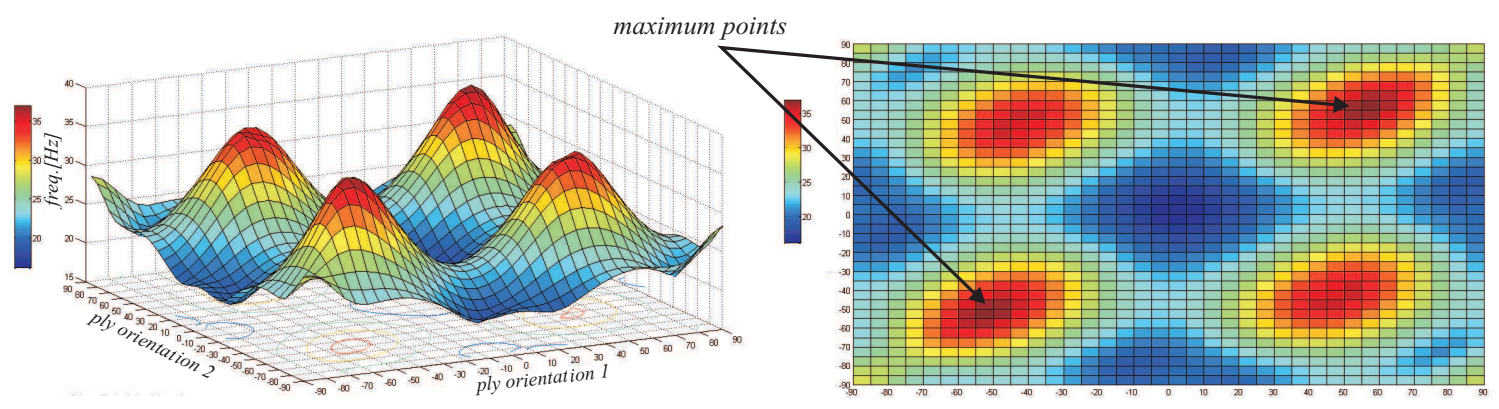

Figure 9. Third structural mode versus fiber orientation.

\subsection{Symmetric Wing}

The next model is a four plies symmetric flat plate wing. The laminated configuration is $[0,90]_{s}$, the material properties are the same shown in table1 The flutter onset occurs in this model for airstream speed around $25.69[\mathrm{~m} / \mathrm{s}]$, and again the mode responsible for the flutter effects onset is the second structural mode, the first torsional mode. The vgf plots (figure 10) shows the wing performance at a given airstream range.

After the initial analysis, the optimization algorithm chooses the second eigenvalue and their associated first torsional mode as the optimization targets. The results are shown in table 3 ,

Table 2. Summarize of optimized results.

\begin{tabular}{ccc}
\hline & Initial Configuration & Optimized Configuration \\
\hline Ply 1 orientation [degrees] & 0 & 53.83 \\
\hline Ply 2 orientation [degrees] & 90 & 53.83 \\
\hline First torsional freq. $[\mathrm{Hz}]$ & 16.57 & 36.95 \\
\hline Flutter speed onset $[\mathrm{m} / \mathrm{s}]$ & 9.2 & 19.96 \\
\hline
\end{tabular}




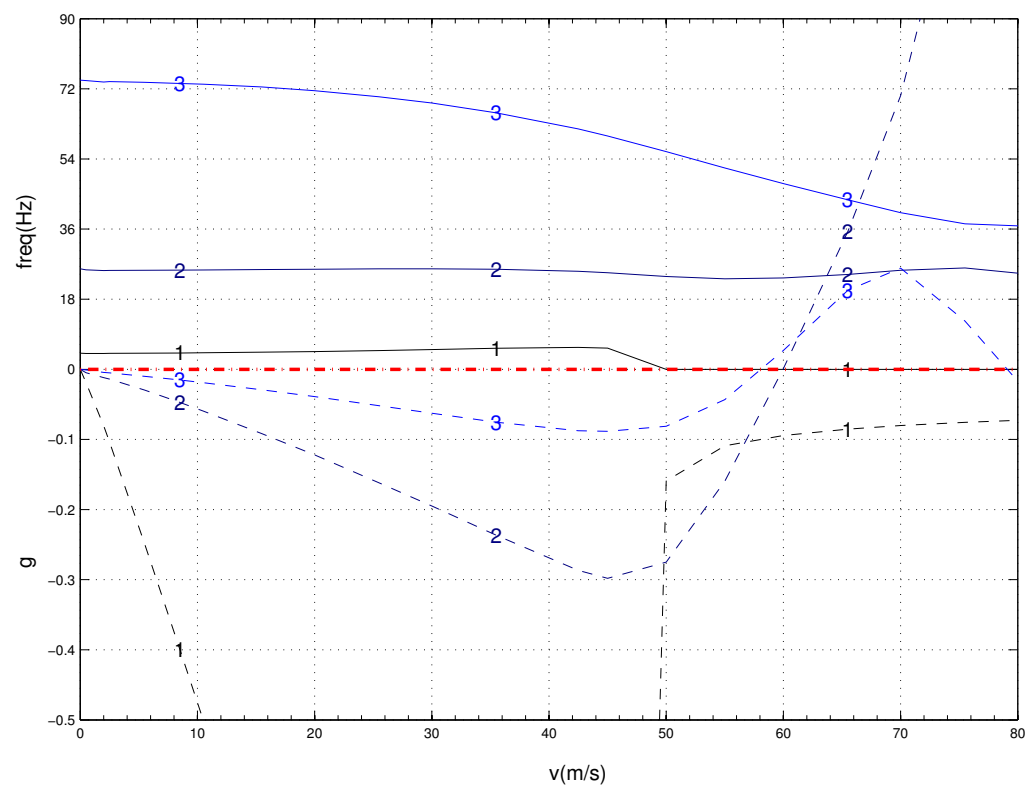

Figure 10. The VGF plot of the symmetric plate.

Table 3. Summarize of optimized results.

\begin{tabular}{ccc}
\hline & Initial Configuration & Optimized Configuration \\
\hline Ply 1 orientation [degrees] & 0 & -52.50 \\
\hline Ply 2 orientation [degrees] & 90 & 44.30 \\
\hline Ply 3 orientation [degrees] & 90 & 44.30 \\
\hline Ply 4 orientation [degrees] & 0 & -52.50 \\
\hline Flutter speed onset $[\mathrm{m} / \mathrm{s}$ ] & 25.69 & 58.10 \\
\hline
\end{tabular}

\subsection{Comparison between MAC and simple repeated eigenvalues}

In all cases tested in this work, the eigenvalue related to the target mode reaches the next eigenvalue during the maximization procedure. When it occurs, it is difficult to see clearly which eigenvalue has to be updated by the algorithm. The target mode has two eigenvalues with almost the same value related to it. This problem is known as a root multiplicity problem. As stated before, this work uses the MAC as the tool to deal with this problem, when it is necessary.

The two figures below show a comparison between the optimization evolution of the non-symmetric (11) and the symmetric plate (12) using MAC and using a simpler mechanism that just detects the root multiplicity and solves it by means of the extreme gradients technique. In both cases the MAC procedure converges faster than the extreme gradients technique. In the problems presented in this work computational time is not a big problem, regarding the simplicity of the models, however the results show MAC as a better choice in tracking mode switching problems. 


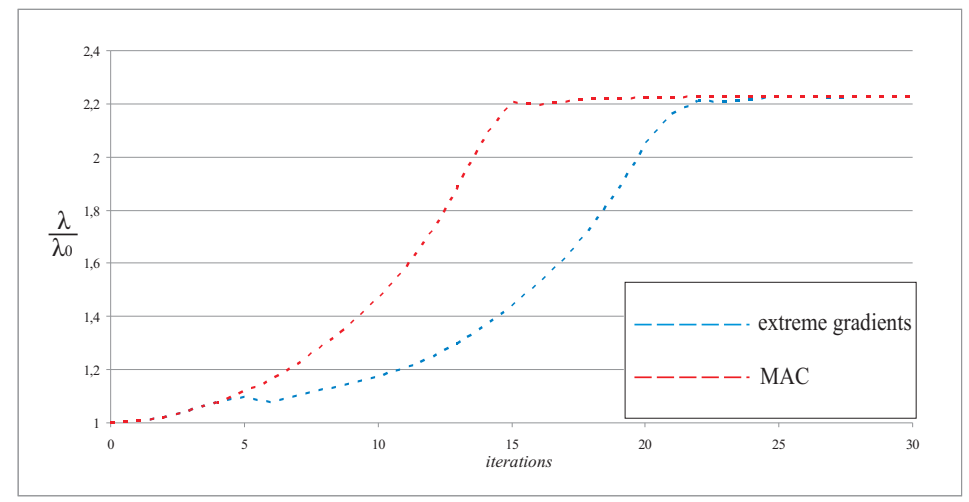

Figure 11. Objective function evolution using MAC and extreme gradients technique.

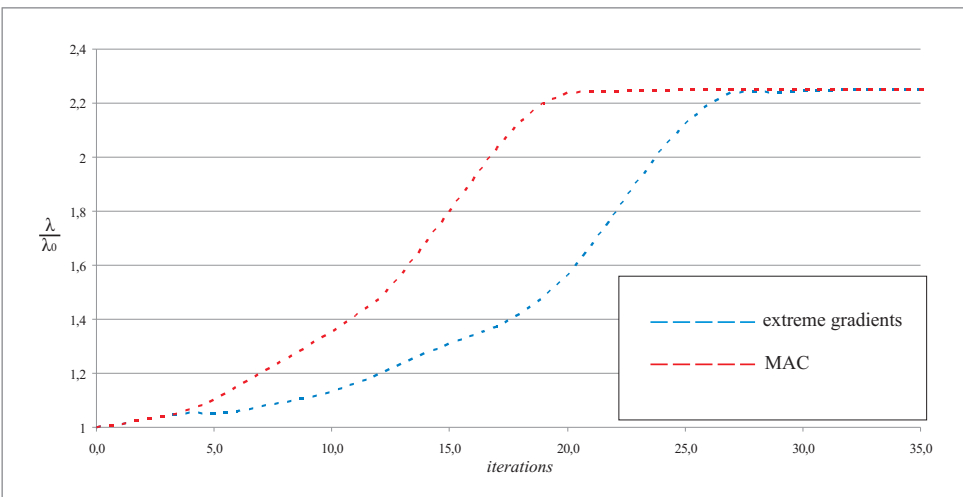

Figure 12. Objective function and MAC for the symmetric plate.

\section{CONCLUSIONS}

The optimization procedure proposed in this work succeeds in improving the aeroelastic behavior of a flat plate composite wing. Regarding the simplicity of the model applied in this work, the assumptions are sufficient to show the possibilities of the methodology. The couple between structural optimization techniques and aeroelastic analysis plays an important role in the design of laminate composite flat-plate wings. The actual advances in laminate manufacturing become these work results affordable choices in aeroelastic design.

The mode tracking switching scheme presented in this work shows better results comparing to simpler mode tracking techniques as the application of extreme gradients. The comparison between MAC and extreme gradients shows the modal criterion as a good and safe choice when a root multiplicity problem has to be handled by the algorithm.

The special attention to the structural optimization in aeroelastic tailoring results is well established in this work. A good formulation of the optimization problem helps to get great results in the aerodynamic features of the composite structures. The appropriated techniques and formulation can turn the search for solution easier and faster.

\section{Acknowledgements}

The authors would like to thanks Capes by the financial support of part of this research. 


\section{REFERENCES}

[1] Albano E., Rodden W. P., "A Doublet-Lattice Method for Calculating Lift Distributions on Oscillating Surfaces in Subsonic Flows". AIAA Journal 7, 279-285, 1969.

[2] Allemang R. J., "The modal assurance criterion - Twenty years of use and abuse", Sound and Vibrations, 14-21, 2003.

[3] Bunton R. W., Denegri Jr. C. M. "Limit Cycle Oscillation Characteristics of Fighter Aircraft". Journal of Aircarft 37(5), 916-918, 2000.

[4] Chen P. C. "Damping Perturbation Method for Flutter Solution: The g-method.". AIAA Journal 38(9), 1519-1524, 2000.

[5] EADS Deutschland GmbH, C. R. C. "The research requirements of the transport sectors to facilitate an increased usage of composite materials, Part 1 : The Composite Material Research Requirements of the Aerospace Industry", 2004.

[6] Giesing J., Kalman, T., Rodden, W., "Subsonic Unstweady Aerodynamics for General Configurations”. Technical report affdl-tr-71-5, Air Force Flight Dynamics Laboratory, 1971.

[7] Harder R. L., Desmarais R. N. "Interpolation Using Surface Splines”, Journal of Aircraft 9, 189-191, 1972.

[8] Hertz T. J., Shirk M. H., Ricketts R. H., Weisshaar, T. A., "Aeroelastic Tailoring with Composites Applied to Forward Swept Wings." Technical report, Flight Dynamics Laboratory (FIBR) AF Wright Aeronautical Laboratories, 1981.

[9] Irvine T., "http://www.vibrationdata.com/tutorials2/ModalMass.pdf”. Web page consulted on October 14th, 2010.

[10] Jones R. M., "Mechanics of Composite Materials". Scripta Book Company, Washington 2nd edition, 1999.

[11] Kim T. S., Kim Y. Y., "Mac-based mode tracking in structural topology optimization", Computers and Structures 74, 375-383, 2000.

[12] Kumar W. P. P., Palaninathan R., "Finite Element Analysis of Laminated Shells with Exact Through-Thickness Integration", Computers and Structures, 63(1), 173-184, 1997.

[13] Reddy J. N. "Mechanics of Composite Plates and Shells - Theory and Analysis". 2nd edition, 2004.

[14] von Gersdorff K., "Bölkow-Sportflugzeuge: Die Sport-, Schul- und Reiseflugzeuge mit Entwürfen und Projektender Firmen Klemm, Bökow, SIAT, MBB, LFU und Mylius von 1956 bis 1979 Buchzentrum Empfingen”, 1981.

[15] Weisshaar T. A., “Aircraft Aeroelastic Design and Analysis”. School of Astronautics and Aeronautics - Purdue University, Class Notes, 1995.

[16] Zhang Y. X., Yang Z. H., "Recent Developments in Finite Element Analysis for Laminated Composite Plates", Composite Structures 88, 147-157, 2009.

[17] Zienkiewicz O., Taylor R. L., "The Finite Element Method - The Basis". Elsevier Academic Press, 5th edition volume 1, 2000.

[18] ZONA-Technology., “ZAERO Theoretical Manual”. ZONA Technology Inc., Scottsdale, Arizona, USA., 15a edition, 2006. 